\title{
Globe
}

Revue internationale d'études québécoises

\section{Bill Marshall : Quebec National Cinema. Montréal et Kingston, McGill-Queen's University Press, 2001}

\section{Jean-Claude Jaubert}

Volume 5, numéro 1, 2002

URI : https://id.erudit.org/iderudit/1000670ar

DOI : https://doi.org/10.7202/1000670ar

Aller au sommaire du numéro

Éditeur(s)

Globe, Revue internationale d'études québécoises

ISSN

1481-5869 (imprimé)

1923-8231 (numérique)

Découvrir la revue

Citer ce compte rendu

Jaubert, J.-C. (2002). Compte rendu de [Bill Marshall : Quebec National Cinema.

Montréal et Kingston, McGill-Queen's University Press, 2001]. Globe, 5(1),

179-183. https://doi.org/10.7202/1000670ar d'utilisation que vous pouvez consulter en ligne.

https://apropos.erudit.org/fr/usagers/politique-dutilisation/ 


\section{Recensions}

\section{Bill Marshall}

Quebec National Cinema.

Montréal et Kingston,

McGill-Queen's University Press, 2001.

Tous ceux qu'intéresse la connaissance approfondie du cinéma québécois francophone depuis les années 1960 trouveront dans le livre de Bill Marshall une somme jamais égalée, à mon avis, en anglais aussi bien qu'en français, de connaissances, d'analyses et de réflexions sur la production francophone des quatre dernières décennies au Québec. Bill Marshall, professeur d'études françaises à l'Université de Glasgow, définit dans sa préface sa recherche comme une approche exhaustive et multidimensionnelle de son sujet. En refermant le livre après une lecture stimulante des 312 pages du texte et des 26 pages de notes, suivies d'une très exhaustive bibliographie, force est de conclure que le but premier de l'auteur a été atteint.

À la différence de la "classique. Histoire générale du cinéma au Québec de Yves Lever, l'étude de Bill Marshall est conduite selon une perspective thématique plutôt qu'historique. L'auteur s'est fixé un cadre de recherche à partir duquel il explore méthodiquement l'essentiel de la production de films de fiction au cours de la période choisie. Ce cadre, c'est celui de l'identité nationale. D'où ce titre intriguant que l'on hésite à traduire par " cinéma national québécois ". L'auteur indique d'emblée que, pour lui, le cinéma qu'il va explorer possède dans son ensemble une identité nationale. Ce faisant, il se garde bien de prendre parti sur la question de la souveraineté et laisse prudemment aux lecteurs le soin de décider à l'issue de l'étude - selon leur conviction propre - si le cinéma québécois dans son ensemble est favorable ou non à la souveraineté du Québec.

Peter Graefe et Michel Lacroix [éd.], * Recensions *, Globe. Revue internationale d'études québécoises, vol. $5, \mathrm{n}^{\circ} 1,2002$. 
Le premier chapitre, comme il se doit, expose le cadre théorique de l'étude. Qu'est-ce qu'un cinéma national ? Reconnaissant qu'il n'existe pas de modèle herméneutique permettant de décider si un film répond ou non à un critère appelé " national s qui n'a pu être défini au préalable, l'auteur fait alors appel à différents théoriciens de l'identité nationale pour établir les critères qui le guideront. Il cite ainsi les quatre éléments fondamentaux qui peuvent servir à évaluer l'identité nationale: "reconnaissance, espace, temps et identité ontologique" (p. 5). Un autre concept sera souvent utilisé au cours des nombreuses et riches analyses de films qui constituent l'essentiel de l'ouvrage, c'est celui de la tension constante qui s'exerce dans les productions artistiques entre les forces centripètes et les forces centrifuges, celles qui éloignent ou rapprochent de l'identité nationale, celles qui "territorialisent ", opposées à celles qui " déterritorialisent . Se méfiant avant tout du piège qui consiste à identifier un cinéma national en recherchant une cohérence et une unité, Marshall cherche au contraire à saisir le cinéma québécois non pas dans sa seule cohérence, mais aussi dans ses manifestations d'incohérence (p. 13). Il explore ainsi comment le cinéma construit une hégémonie nationale toujours provisoire, quelles relations il entretient avec le reste de la culture québécoise et avec l'industrie cinématographique mondiale. La dernière partie de ce chapitre d'introduction présente brièvement le contexte économique et politique de la production et de la diffusion du cinéma québécois au Québec.

À partir du deuxième chapitre, l'étude est organisée par thèmes ou par genres. Marshall commence par étudier deux films qualifiés de "fondateurs ", Pour la suite du monde et $\dot{A}$ tout prendre. Pour ce qui est du premier, il montre que les cinéastes, Perrault et Brault, ont davantage " produit " de la réalité que filmé la réalité, brouillant ainsi la frontière entre documentaire et fiction. Il analyse ce film comme le site même des différentes forces et tendances qui traversent la nation. Alors que le film de Perrault et Brault peut être vu comme une recherche de "l'authenticité " (guillemets de Marshall) derrière l'apparence, Jutra au contraire sape joyeusement le " moi " sur lequel il semble se concentrer de façon narcissique. Avec ce chapitre, nous avons un avant-goût de ce qui fait la force et l'originalité de ce livre : l'analyse méthodique de scènes de film, interprétées par l'auteur d'une manière qui donne toute sa crédibilité au point de vue proposé, point de vue qui s'éloigne parfois beaucoup d'interprétations proposées auparavant par les critiques ou les historiens. Que l'on adopte sans réserve les conclusions de l'auteur ou que l'on 


\section{RECENSIONS}

trouve matière à divergence dans les analyses de Marshall, on ne peut de toute façon que reconnaître la qualité de la documentation. Non seulement les films traités représentent la très large majorité de la production québécoise de la période, mais leur analyse est appuyée sur la lecture d'une très abondante somme d'analyses et d'études portant sur les films eux-mêmes, ainsi que par une très solide documentation générale sur l'histoire politique et sociale du Québec.

Une fois que ces "fictions fondamentales " ont été analysées, Marshall élargit le champ d'étude et aborde au chapitre suivant l'ensemble de la production de fiction des années 1960, production qu'il désigne globalement comme un "cinéma de la modernisation ", modernisation du pays s'entend. Les deux concepts fondamentaux qui guident cette fois l'étude des films de cette période sont les concepts de modernisation et d'hégémonie. La modernisation est ici définie comme le processus qui permet à une économie et à une société de s'aligner sur les exigences du capital, processus caractérisé par la rapide transformation des pratiques traditionnelles, des communautés, des croyances et des identités (p. 47). Quant à l'hégémonie, elle représente les valeurs et les idées de la classe dominante dans la société. Analysant le film de Groulx, Le chat dans le sac, l'auteur montre que les tensions qui sous-tendent l'action des personnages peuvent se voir comme les manifestions des obstacles et des contradictions qui accompagnent tout processus de modernisation et d'élaboration d'une hégémonie. Poursuivant son étude des films de cette décennie fondatrice, Marshall analyse avec la même pertinence les films de Lefebvre, de Patry, de Carle, de Brault, etc.

Constatant l'abondance des références à la France dans la culture populaire québécoise, Marshall consacre son quatrième chapitre à l'ambivalence de ces références dans de nombreux films québécois des années 1960 et 1970 . À la différence de la thèse du "roman familial" défendue par Heinz Winneman dans Cinéma de l'imaginaire québécois : de la Petite Aurore à Jésus de Montréal, Marshall se situe davantage sur un terrain historique et concret. Puis, dans le chapitre "Sex and the Nation . Marshall étudie les très nombreux films québécois qui placent les relations familiales au cœur de leur problématique. Il s'appuie largement pour ce faire sur des concepts freudiens. Les analyses de films fondamentaux dans ce domaine, comme Le temps d'une chasse, Les bons débarras, Un Zoo la nuit et Léolo, vont bien au-delà de la quête ou réconciliation/rejet du père. Il est logique de traiter dans ce chapitre la 
question de l'homosexualité qui apparaît en filigrane dans de nombreux films. Pour l'auteur, la représentation de l'homosexualité dans les films québécois pourrait se comparer à la représentation de l'immigrant ou de l'autochtone (ce sujet spécifique fera l'objet des chapitres 9 et 10) dans la mesure où elle témoigne de la capacité d'une nation à se montrer inclusive plutôt qu'exclusive.

Peut-on parler d'un " cinéma d'auteur " au Québec? Le chapitre traitant de cette question est en réalité plus large que ne le laisse supposer l'énoncé de son titre. L'auteur présente d'abord le contexte économique de la production québécoise, avant de se demander si le concept de cinéma d'auteur tel qu'il est formulé au début des années 1960 par les critiques français peut s'appliquer au Québec. Bien sûr, il y a l'exemple des cinéastes reconnus de la première génération, les Jutra, Carle, Arcand, Lefebvre, mais il conclut que ce concept s'adapte mal à la réalité québécoise. Cinéma d'auteur, cinéma populaire, cinéma de qualité sont des termes qui peuvent s'appliquer au même film, comme par exemple au Déclin de l'empire américain ou à Mon oncle Antoine. En fonction du point de vue inclusif adopté délibérément par Bill Marshall, les films dits "populaires" ont autant d'importance pour l'auteur que les films "de qualité ", traditionnellement privilégiés par les historiens et les critiques de cinéma. Le chapitre consacré au cinéma populaire fait donc une large place à des films habituellement mentionnés uniquement pour mémoire dans d'autres ouvrages. D'ailleurs, ce type de film aurait davantage tendance à traiter la question de la nation confrontée à la mondialisation en s'appuyant sur une culture nord-américaine présentée comme la pierre de touche du succès et de la mobilité.

Comme tous les autres chapitres, le chapitre consacré au cinéma des femmes commence par une présentation très bien informée du contexte historique et social. Marshall est bien conscient qu'un nombre non négligeable de réalisatrices considèrent aujourd'hui que différencier le cinéma des femmes du cinéma des hommes revient à enfermer ces premières dans un ghetto; il estime cependant que le cinéma des hommes est tellement marqué par le genre au Québec et si peu inclusif du "féminin ", qu'il lui paraît normal de consacrer une part de son étude au cinéma des femmes.

Une étude qui a pour fil directeur principal l'identité nationale dans le cinéma ne pouvait être complète sans accorder une place importante 
à la fois à la question autochtone et à celle de l'immigration. Bien qu'il traite dans ces deux parties des films et des auteurs essentiels sur ces deux questions, il faut admettre que ce ne sont pas les chapitres les plus complets ni les plus incisifs sur le sujet. Marshall consacre la dernière partie de son étude à la question de l'opposition modernité/postmodernité et, bien qu'il s'en défende, ce chapitre est un peu le point culminant de sa quête de l'identité nationale dans le cinéma québécois. Il y souligne par exemple la place que tient la métropole de Montréal dans ce cinéma, ville qui est au cœur de la tension allégorique nationale dans la culture québécoise et dont la présence s'impose dans une grande quantité de films de la toute nouvelle génération de cinéastes québécois. Estimant - à juste titre - que la question essentielle pour le cinéma québécois est de conserver le sens du passé qui fait sa différence dans sa façon de traduire allégoriquement la tension nationale, c'est dans les films de Robert Lepage qu'il trouve le meilleur exemple d'une synergie établie entre le passé et le présent. Un livse tel que celui de Bill Marshall ne se lit pas en quelques heures. Il renferme une telle somme d'informations, appuyées par un raisonnement théorique, que l'on doit le prendre par doses raisonnables, mais l'effort du lecteur est largement récompensé par la stimulation que procure sa lecture. En outre, on peut également l'utiliser comme livre de référence grâce à l'index des noms et des titres qui termine l'ouvrage.

Jean-Claude Jaubert

Collège universitaire Glendon (Canada)

\section{Michel Biron}

L'absence du maître.

Saint-Denys Garneau, Ferron, Ducharme.

Montréal, Presses de l'Université de Montréal, coll. "Socius ", 2000.

Ce qui retient d'abord l'attention dans ce nouvel essai de sociocritique consacré à la littérature québécoise est l'exceptionnelle souplesse d'analyse de Michel Biron. Spécialiste de la littérature belge, à laquelle il a consacré un premier ouvrage en 1994, La modernité belge. Texte et société, Biron démontre avec L'absence du maître que les littératures 